
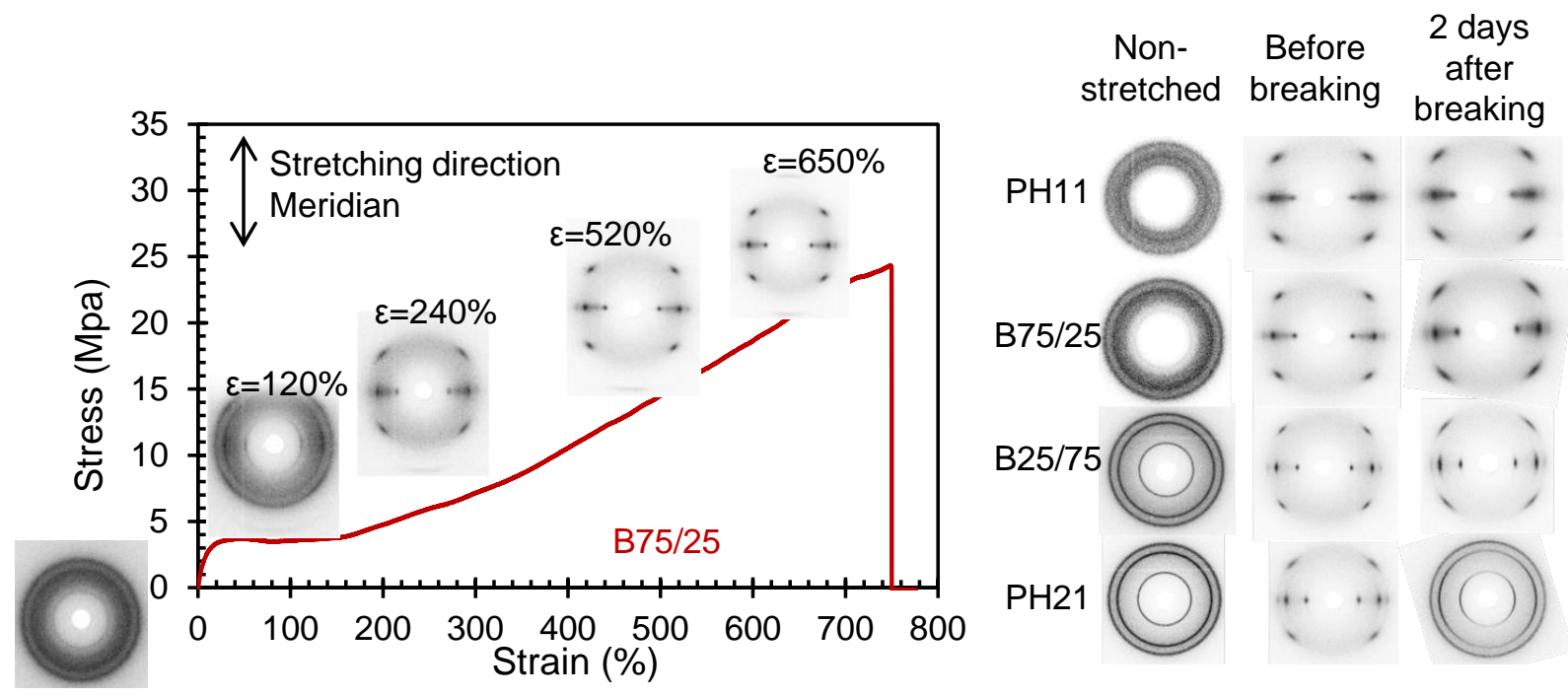


\title{
Mechanical properties and stress-induced polymorphic transformations of blends of random isotactic propylene-1-hexene copolymers
}

\author{
Hamed Janani, Rufina G. Alamo* \\ FAMU-FSU College of Engineering, Department of Chemical and Biomedical Engineering
}

2525 Pottsdamer St. Tallahassee, FL 32310

\begin{abstract}
:
The mechanical properties of binary blends of propylene-1-hexene random copolymers (with 11 and $21 \mathrm{~mol} \%$ 1-hexene) are studied in parallel with polymorphic transformations under uniaxial tensile deformation. The modulus, yield stress, and draw ratio of the pure PH copolymers decrease with increasing 1-hexene content, while for the blends the change of mechanical properties with composition is highly non-linear. The addition of just $10 \mathrm{wt} \%$ PH11 to PH21 doubles the elastic modulus and yield strength of the blends in reference to the value of PH21, reaching for all blends values close to the performance of pure PH11. The elongation at break and the ultimate tensile strength increase more gradually with content of PH11. On tensile deformation, pure components and blends undergo morphological and polymorphic transformations, such as a reversible lamellar to fibrillar transformation of trigonal PH21, or an irreversible $\alpha$ crystal to mesophase in pure PH11 and the blends. In blends and neat PH11, a fibrillar trigonal morphology that develops under deformation is stabilized by the transformation of $\alpha$ to mesophase, and remains after removing the load, explaining the lower elastic recovery of the blends compared to PH21. The formation of stress-induced trigonal crystallites in PH11 and blends after strains $>150 \%$ is explained as a decrease of the free energy barrier for nucleation of a phase that requires short iPP sequences.
\end{abstract}

\footnotetext{
* Corresponding author
} 


\section{Introduction:}

Incorporation of stereo defects and low molecular weight $\alpha$-olefins such as ethylene and 1-butene in the chain of isotactic polypropylene (iPP), using metallocene catalysts, has been effectively utilized to change the mechanical properties of iPP from a rigid thermoplastic to elastoplastomeric and to elastomeric behavior [1-10]. Adding defects or a comonomer to the iPP macromolecule decreases the crystallizable sequence length in the chain, and hence the degree of crystallinity decreases. The iPP polymorphic behavior also changes with type and defect content as amply studied in the last two decades [11-24]. Conversely, blending different types of iPPs, iPP with iPP-based copolymers, or blending different iPP-based copolymers is also an avenue for engineering mechanical properties. Compared to the synthetic approach, iPP blends are often a cost-effective path to change the material properties. Blends of propylene-ethylene (PE) and propylene-1-butene (PB) copolymers have been studied extensively, and some of these blends are produced commercially for packaging applications. [25,26] However, the mechanical behavior of blends of iPP copolymers based on higher $\alpha$-olefin comonomers such as 1 -hexene and 1-octene is less known [27,28].

Recently, we reported a study of melt miscibility, crystallization kinetics and polymorphic behavior of binary blends of isotactic propylene and 1-hexene random copolymers with 11 (PH11) and 21 (PH21) mol\% 1-hexene [29,30]. Blends of iPP and PH11, and blends of PH11 and PH21 are miscible in the melt, while blends of iPP and PH21 are inmiscible. Furthermore, a detailed study of the crystallization kinetics of blends of PH11 and PH21 revealed that the blends form two populations of crystallites. The PH11 component crystallizes in a first stage in the monoclinic phase with the 1-hexene co-units rejected from the crystallites. PH21 crystallizes in a second stage in trigonal crystallites with the 1-hexene counit participating in the trigonal unit cell. Due to the rejection of the 1-hexene counit in the first crystallization stage, the crystallization kinetics of trigonal crystals in the blends increase in reference to the crystallization kinetics of pure $\mathrm{PH} 21$ [8]. In the present work, we expand these studies to investigating the mechanical properties of blends of these two $\mathrm{PH}$ copolymers which as individual constituents form different crystallographic phases, monoclinic $(\alpha)$ for PH11 and trigonal ( $\delta)$ in $\mathrm{PH} 21$. 
The mechanical properties of neat PH copolymers have been studied as a function of content of 1-hexene up to $\sim 26 \mathrm{~mol} \%[31,32,33,19]$. With increasing content of 1-hexene, the level of crystallinity decreases, accordingly, the modulus and yield stress decrease while tensile elongation increases, the necking region becomes more diffuse [32], and the impact strength is significantly improved [33]. It was also observed that the yield stress decreases logarithmically with 1-hexene for $\mathrm{PH}$ with $<10 \mathrm{~mol} \%$ 1-hexene [19], a behavior also observed in propylene 1octene copolymers (PO) [19]. Even at low contents of 1-hexene ( $\sim 1 \mathrm{~mol} \%)$, PH copolymers are thermoplastic materials with high flexibility [32]. In the range of $15-30 \mathrm{~mol} \%$, $\mathrm{PH}$ copolymers crystallize in the trigonal phase which changes significantly their mechanical properties; the strain hardening and tensile strength decrease compared to copolymers with lower comonomer content [32]. Due to the formation of trigonal crystals, in PH copolymers with $>13$ mol\% 1 hexene, the yield stress is higher than for PO copolymers with matched comonomer content [19].

De Rosa et al. carried out a parallel study of the tensile behavior and strain-induced polymorphic transformations of $\mathrm{PH}$ copolymers under uniaxial deformation [32]. For copolymers with 1hexene content $<9 \mathrm{~mol} \%$, a transformation of $\alpha$ to mesophase was observed at relatively low elongations (100-200\%). They explained that mechanical melting of the original $\alpha$ crystals was followed by reorganization of the chains that are pulled out of the lamellae during mechanical melting into mesomorphic longitudinally oriented bundles with low lateral order [32]. Copolymers with 1-hexene $>9 \mathrm{~mol} \%$ behave differently in terms of structural transformations under tensile deformation. For these copolymers the characteristic reflections of the $\alpha$ phase disappear during deformation and reflections of the trigonal phase appear in addition to reflections associated with the mesophase. It was then concluded that the initial $\alpha$ phase crystals transform partly into trigonal and partly into mesophase for these copolymers [32]. No polymorphic transformation during deformation was observed for PH copolymers with 1-hexene content higher than $18 \mathrm{~mol} \%$ which originally crystallize in the trigonal phase [32].

Unlike monoclinic crystallites, PH trigonal crystallites orient but do not transform to the mesophase under elongation, therefore, it was concluded that the trigonal phase was more stable under deformation [31]. On this basis, the formation of trigonal crystallites in PH11/PH21 blends, even at low levels of PH21 should influence their mechanical properties. Trigonal crystals that develop between the lamellae of monoclinic crystallites in the blends are expected to 
reinforce the mechanical strength making the materials more resilient to deformation. This behavior is tested in the present work by analyzing the mechanical properties under tensile deformation of PH1 1/PH21 blends, and collecting parallel 2D WAXD patterns to follow possible crystal transformations in the whole range of blend composition.

\section{Experimental Section:}

Materials: The isotactic propylene 1-hexene random copolymers used, PH11 and PH21, are experimental samples synthesized by ExxonMobil using a single-site metallocene catalyst. The copolymers have 11 and $21 \mathrm{~mol} \% 1$-hexene, a $\mathrm{Mw}=160 \mathrm{~K}+/-5 \mathrm{~K} \mathrm{~g} / \mathrm{mol}$ and $\mathrm{PDI}=2.03$ as described in prior works $[11,29]$. Blends of $\mathrm{PH} 11 / \mathrm{PH} 21$ were prepared in solution dissolving weighed proportional masses of each component in xylene at $125 \pm 1{ }^{\circ} \mathrm{C}$ in a concentration of $0.5 \mathrm{~g}$ blend $/ 100 \mathrm{ml}$ solvent and stirred for $15 \mathrm{~min}$. The solution was further poured in methanol at $-10{ }^{\circ} \mathrm{C}$. The crystals were filtered, washed with methanol and dried overnight at $60{ }^{\circ} \mathrm{C}$ in vacuum until constant mass. The pure components were also subjected to the same solvent procedure. Blends are labeled by B followed by mass percent of PH11/mass percent of PH21. For example, B25/75 is the blend with $25 \%$ by mass of PH11 and $75 \%$ PH21. Films, $\sim 250 \mu \mathrm{m}$ thick, of each blend were prepared by melt compression molding at $200^{\circ} \mathrm{C}$ and quenched to room temperature $\left(23 \pm 1^{\circ} \mathrm{C}\right)$. The films were kept at room temperature for at least two weeks prior to any testing.

Stress-strain measurements: Dumbbell shape samples were cut from the original films with a neck width of $2 \mathrm{~mm}$, gauge length of $5.5 \mathrm{~mm}$ and thickness of $0.25 \mathrm{~mm}$. Stress-strain curves were obtained at a drawing rate of $25 \mathrm{~mm} / \mathrm{min}$ using a Thümler tensile test machine model $\mathrm{TH}$ 2730. Two bench marks were drawn in the neck region to follow the elongation. The engineering strain $(\varepsilon)$ was obtained as $\varepsilon=\left(l-l_{0}\right) / l_{0}$ with $l_{0}$ as the initial marked gauge length and $l$ the distance between marks during deformation. The engineering stress $(\sigma)$ is the force per initial cross sectional area. The elastic modulus (E) was obtained from the slope of the linear region at low deformation. The yield stress, $\sigma_{\mathrm{y}}$, is taken at the point where $\frac{d q}{d z} \approx 0$ after the region of linear deformation. The tensile strength, $\sigma \mathrm{b}$, and elongation at break, $\varepsilon$ b, were obtained from the values of stress and strain at breaking point. The intensity of strain-hardening was characterized by the slope of the stress-strain region at high elongations, those close to break, and by the difference 
between tensile and yield strengths, $\sigma_{b}-\sigma_{\mathrm{y}}$. The recovered strain after break, $r_{\mathrm{b}}$, was also recorded as it is a measure of the elastomeric behavior of pure components and blends. $r b$ was measured about two weeks after break as, $\left(l_{b}-l_{\mathrm{f}}\right) / l_{\mathrm{b}}$ where $l_{\mathrm{b}}$ is the length of the specimen at break and $l_{\mathrm{f}}$ the length after break. All reported values are averages over five independent stress-strain experiments for each sample.

Polymorphic transformations under tensile deformation: To study changes in crystal structure under deformation, a house-made stretching device was built to be able to deform the copolymers and blends inside the sample compartment of the Bruker Nanostar diffractometer. The dumbbell-shaped sample was stretched to a specific strain and WAXD and SAXS collected simultaneously while under tension. In order to reduce the effect of air scattering, vacuum of $\sim 3$ mbar was applied to the sample compartment. After collecting the patterns, the vacuum was released and the WAXD plate (Fuji Film image plate) was scanned using a Fuji FLA-7000 scanner to obtain the 2D WAXD pattern. The sample was stretched then to a higher strain and this procedure continued up to the breaking strain of the sample.

DSC Melting: The melting of non-stretched samples, aged two weeks at room temperature was obtained using a TA DSC Q2000 instrument connected to an intercooler. The instrument operates under dry nitrogen and was calibrated for temperature and heat flow with indium. The aged samples were heated from -50 to $200^{\circ} \mathrm{C}$ at the rate of $10^{\circ} \mathrm{C} / \mathrm{min}$. Melting was also obtained for stretched samples after breaking in order to study thermal stability of stress-induced crystallites. For the latter, the fibrous region close to the breaking point was encapsulated to record melting also at a heating rate of $10^{\circ} \mathrm{C} / \mathrm{min}$.

\section{Results and Discussion}

Structural analysis of undeformed blends: Prior to the study of the tensile behavior, a brief analysis of the lamellar morphology of the initial non-stretched copolymers and blends is useful for the subsequent discussion of mechanical properties-structure relations. Blends of PH11/PH21 are miscible in the melt in the whole range of composition; however, each component crystallizes separately due to large differences in crystallization kinetics and to the formation of different crystal structures [29,30]. PH11 crystallizes in the monoclinic, $\alpha$ phase, while PH21 crystallizes in the trigonal $\delta$ phase $[14,20,30-33]$. Consequently, the overall heat of melting and 
degree of crystallinity scale linearly with blend composition [29,30]. The distribution and thicknesses of $\alpha$ and $\delta$ lamellae of the specimens aged at room temperature were obtained from background- and Lorentz-corrected SAXS patterns shown in Figure 1a. The SAXS peak of pure PH11 and PH11-rich blends is broad and becomes sharper shifting to lower values of $q$ with increasing content of $\mathrm{PH} 21$. The $q$ shift indicates an increasing long period or increasing average distance between lamellae with increasing content of PH21. The SAXS patterns were further analyzed with the one-dimensional correlation function model for lamellar stacks to extract the long period $(L)$ and the thickness of the crystalline lamellae (lc) and amorphous region (la) [34]. The long period of PH11 and PH21 correspond to 11.2 and $19.2 \mathrm{~nm}$ respectively, in agreement with the values obtained by Poon et al. for similar PH copolymers [19]. It is known that while the long spacing of $\mathrm{PH}$ copolymers decreases with increasing comonomer content up to $11 \mathrm{~mol} \%$, for contents of 1-hexene between 11 and $29 \mathrm{~mol} \%$ the long periodicity increases due to the formation of trigonal crystals $[19,32]$. On the basis of prior morphological and lamellar thicknesses measurements by AFM, the crystal thicknesses extracted from the correlation function for PH11 and PH21 are 6.1 and $11.3 \mathrm{~nm}$ respectively in good agreement with literature data [19].

The lamellar morphology of the PH11/PH21 blends may be separated stacks of $\alpha$ and $\delta$ crystallites or a mixed and intercalated lamellar morphology. In the first case we expect two SAXS peaks at $q$ values close to the value for the pure components, while for mixed lamellae crystals one SAXS peak is expected at intermediate $q$. The SAXS peaks of Figure 1a become broader with increasing content of PH11, but there are single peaked in support of the mixed lamellae morphology. Average data for long spacing and crystal thicknesses are shown to decrease proportionally to the content of PH11 in the blends (Figure 1c). 

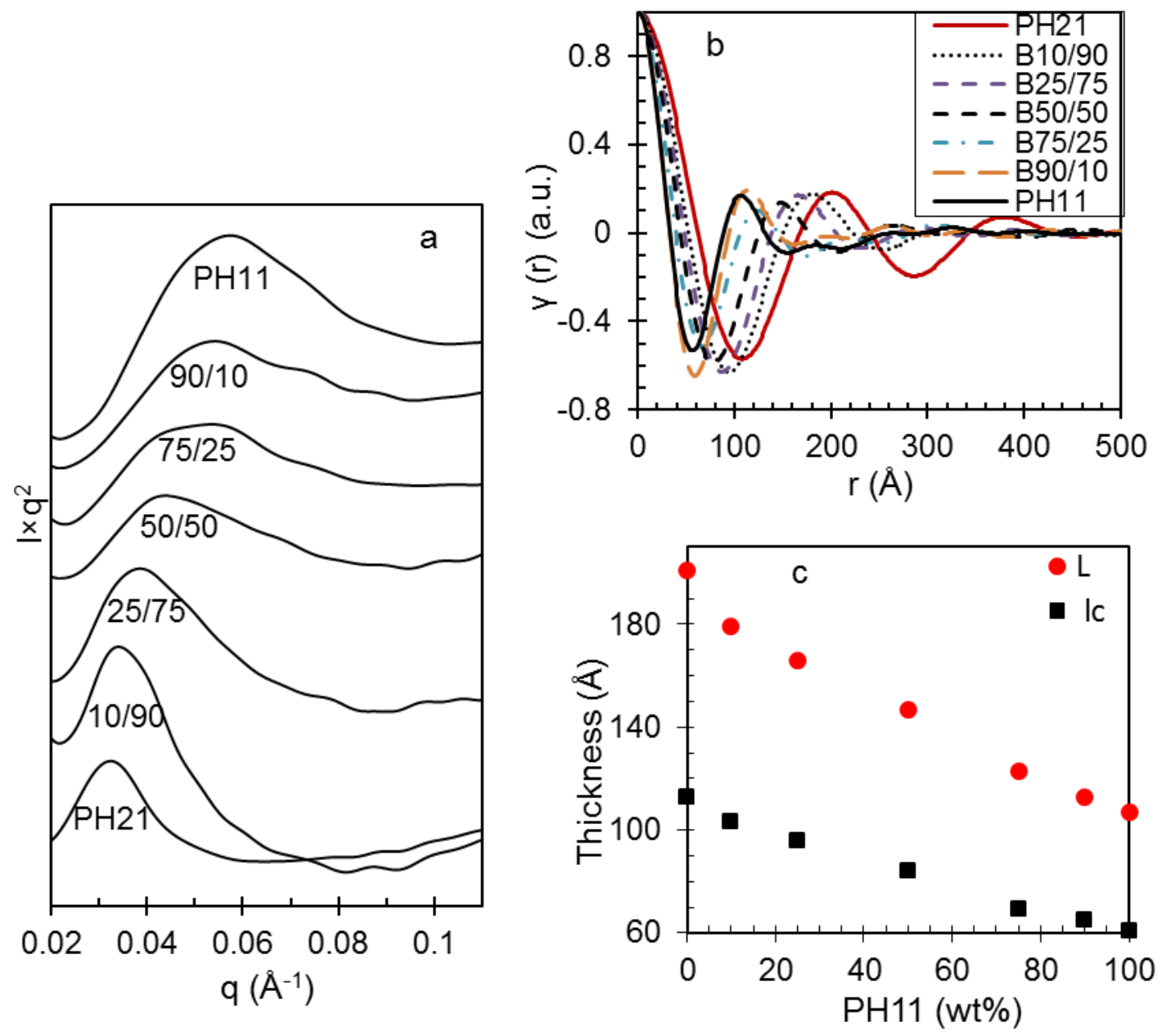

Figure 1. (a) Lorentz-corrected SAXS patterns of PH11/PH21 blends crystallized at room temperature (b) Analysis of (a) with the correlation function (c) change of long spacing and lamellar thickness obtained from the correlation function with increasing content of PH11.

Mechanical properties: The stress-strain curves of PH11/PH21 blends and the pure components under tensile deformation are shown in Figure 2. The pure components and their blends display ductile behavior. Each curve consists of four distinct regions as shown in Figure 2: elastic region, yield point, plastic region (or region of strain softening, $\frac{d \sigma}{d z} \mathbb{E}$ ), and strain hardening region. Mechanical properties including the elastic modulus $E$, yield stress $\sigma_{\mathrm{y}}$, ultimate tensile strength $\sigma_{b}$, elongation at break $\varepsilon$, and percentage of strain recovery after break, $r_{b}$ are listed in Table 1 . 


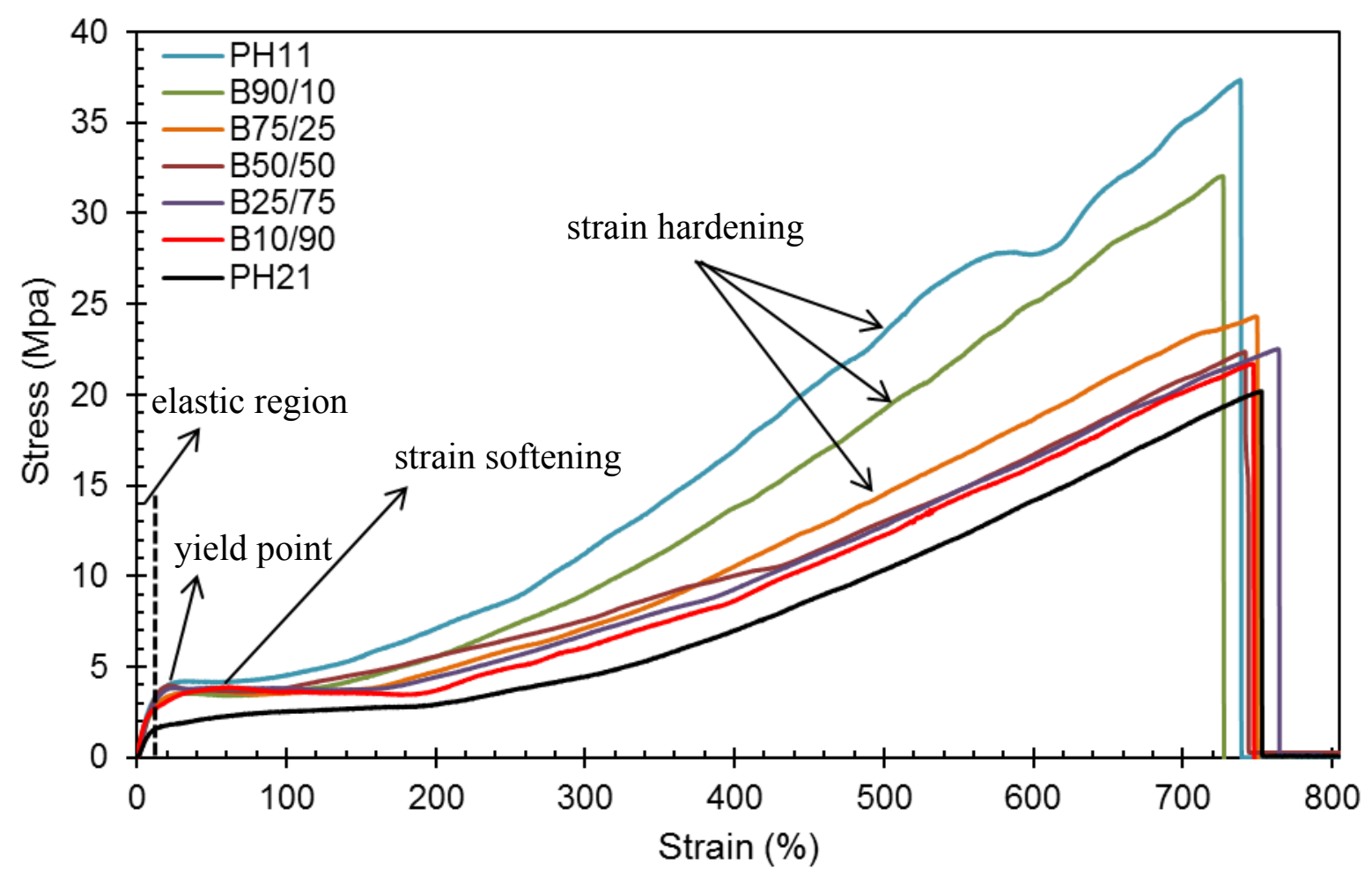

Figure 2. Stress-strain curves for PH11/PH21 blends. The dashed line separates the elastic region. See Figure SI.1 for an expanded region of yield and plastic deformation.

Table 1. Mechanical properties obtained from stress-strain curves of Figure 2.

\begin{tabular}{cccccccc}
\hline & Composition & $\begin{array}{c}\text { Crystallinity } \\
\text { (from } \\
\text { WAXD) }\end{array}$ & $\begin{array}{c}\text { Elastic } \\
\text { Modulus } \\
\text { (Mpa) }\end{array}$ & $\begin{array}{c}\text { Yield } \\
\text { stress } \\
\text { (Mpa) }\end{array}$ & $\begin{array}{c}\text { Stress at } \\
\text { break } \\
\text { (Mpa) }\end{array}$ & $\begin{array}{c}\text { Strain } \\
\text { at } \\
\text { break } \\
\mathbf{( \% )}\end{array}$ & $\begin{array}{c}\text { Strain } \\
\text { recovery } \\
\text { after } \\
\text { break } \\
\text { (\%) }\end{array}$ \\
\hline & $0 / 100$ & 0.24 & $22 \pm 2$ & $2 \pm 0.3$ & $21 \pm 2$ & $650 \pm 95$ & 282 \\
& $10 / 90$ & 0.23 & $45 \pm 3$ & $4.1 \pm 0.6$ & $22 \pm 2$ & $713 \pm 35$ & 204 \\
& $25 / 75$ & 0.22 & $39 \pm 2.5$ & $3.4 \pm 0.8$ & $23 \pm 2$ & $761 \pm 40$ & 186 \\
& $50 / 50$ & 0.20 & $41 \pm 0.5$ & $3.9 \pm 0.3$ & $25 \pm 5$ & $766 \pm 50$ & -- \\
& $75 / 25$ & 0.19 & $42 \pm 3$ & $3.7 \pm 0.1$ & $22 \pm 4$ & $761 \pm 25$ & 117 \\
& $90 / 10$ & 0.17 & $34 \pm 2$ & $3.7 \pm 0.7$ & $32 \pm 1$ & $753 \pm 65$ & --- \\
& $100 / 0$ & 0.17 & $38 \pm 4$ & $3.9 \pm 0.5$ & $34 \pm 6$ & $770 \pm 62$ & 130 \\
\hline
\end{tabular}


In the elastic region the deformation is reversible and the lamellar structure remains intact [3537]. Therefore, it is expected that the elastic modulus is mainly influenced by the content and the nature of the interlamellar region of semicrystalline polymers. The topology of the interlamellar region depends on level of crystallinity, which as seen in Table 1, is not very different for the pure PH11 and PH21 copolymers and their blends [29,30]. As shown in Figure 1, the average lamellae thickness decreases from 11.3 to $6.1 \mathrm{~nm}$ with increasing PH11 and the amorphous thickness, or interlamellar distance defined as $L-I c$ scales linearly between 4.5 for pure PH11 to $8.8 \mathrm{~nm}$ for PH21. The elastic modulus of the blends is plotted as a function of the interlamellar thickness in Figure 3a. The modulus is basically independent of blend composition with values around the modulus of PH11, 45 +/- $5 \mathrm{MPa}$, indicating that due to the mixed lamellar morphology, even small contents of the $\alpha$ phase in the blends serve as reinforcement of the elastic region. Furthermore, it is likely that in the blends molecules of one component interconnect lamellae of the $\alpha$ and $\delta$ phases, for example, via participation of short isotactic sequences of PH11 in the lamellae of PH21. This type of co-crystallization will bring lamellar crystals of PH11 and PH21 closer in the stack. In PH21-rich blends, lamellae insertion of PH11 within the PH21 lamellae act as additional physical crosslinks, thus explaining the relatively high $E$ values for these blends.

The yield stress of semicrystalline polymers has been associated with structural changes of the crystal phase, and explained either by a melt-recrystallization mechanism [38 - 41], by the dislocation theory [42], or by a combination of both processes [43]. In the former, melting occurs in response to local stresses transferred to the lamellae. Miyoshi et al. [44], using in-situ DQ NMR under deformation of ${ }^{13} \mathrm{C}$-labled iPP, reported a reduction in the average number of adjacent re-entry of ${ }^{13} \mathrm{C}$-labled chains with increasing deformation. They explained that the mechanism of mechanical melting includes a chain unfolding process within the lamellae facilitated by helical jump motions of the stems. The dislocation theory, however, attributes the yielding behavior to lamellae fragmentation via sliding or tilting of lamellae stems activated by crystallographic slip motions [45,42]. Using experimental data, a close relationship was found between the yielding behavior and the crystallinity level of polyethylenes $[35,42,46]$. Linear polyethylenes of molar mass $>40,000 \mathrm{~g} / \mathrm{mol}$ usually show a well-defined yield point [35]. As the content of comonomer increases in ethylene 1-alkene copolymers, the yield stress decreases in parallel to the decrease of the degree of crystallinity, and the yield point becomes more diffuse 
[46]. The transition from yielding to plastic deformation of ethylene 1-octene copolymers has been shown to take place at increasingly larger strains with increasing comonomer content, and has been associated with a weaker coupling of lamellar stacks in very low crystallinity random ethylene copolymers [47].
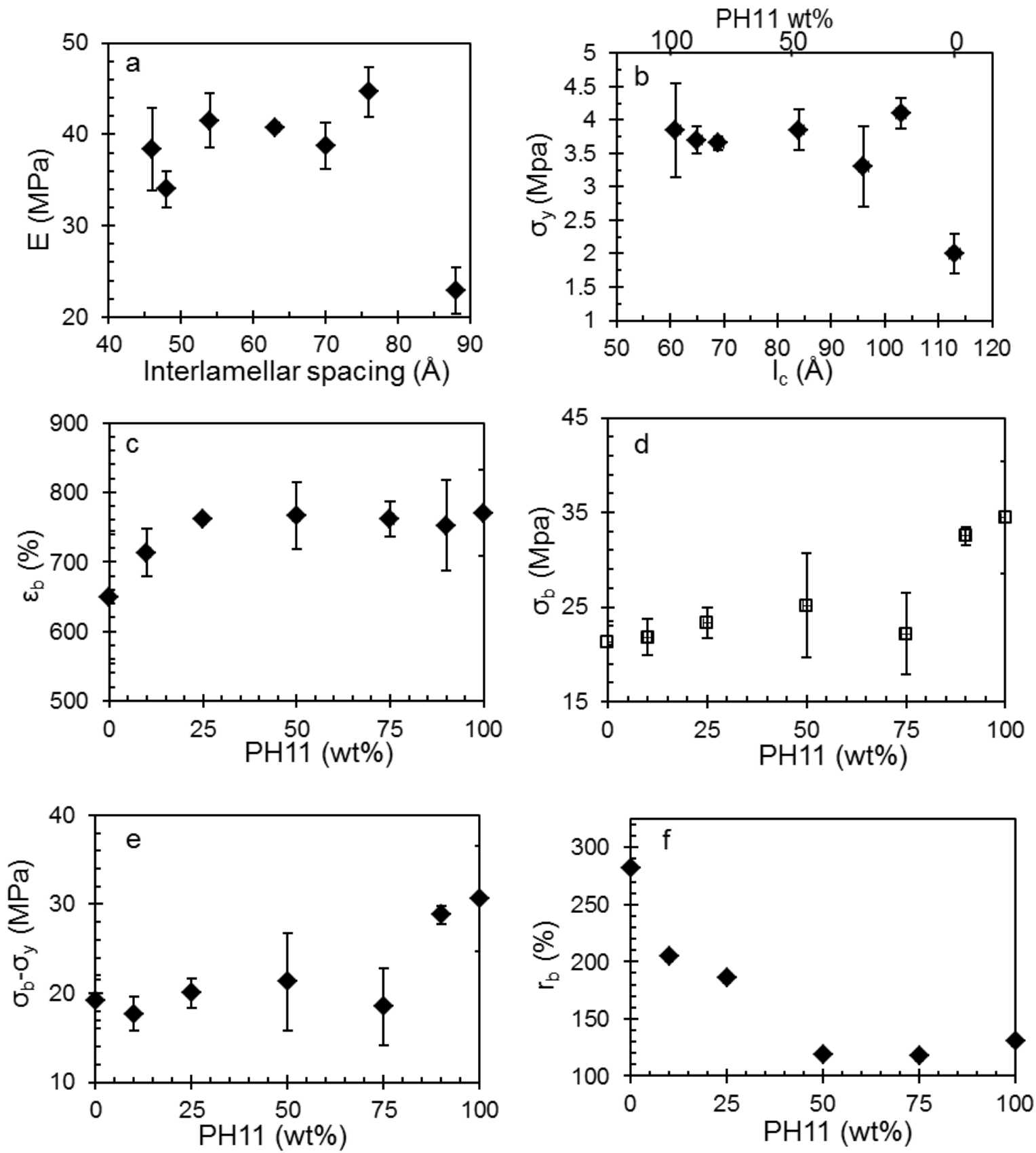

Figure 3. (a) Elastic modulus vs. interlamellar spacing. (b) Yield stress vs. content of PH11 in the blends and vs. average lamellae thickness. (c), (d) Ultimate tensile strain $\left(\varepsilon_{\mathrm{b}}\right)$ and ultimate tensile stress $\left(\sigma_{\mathrm{b}}\right)$ as a 
function of increasing content of PH11. (e) Strain-hardening evaluated as $\sigma_{b}-\sigma_{y}$ vs. content of PH11. (f) Elastic recovery after break as a function of content of PH11.

The average value of the stress at yield for the blends and pure components is plotted in Figure $3 \mathrm{~b}$ against the content of $\mathrm{PH} 11$, and as a function of the average lamellae thickness. The measured yield stress for the pure PH11 and PH21 (3.9 and 2 MPa respectively) are in close agreement with previously reported yield stress of PH copolymers with 11.2 and 18 mol\% 1hexene (4 and 2.5 MPa respectively) [32]. The variation of the yield stress for the blends follows closely the trend of the elastic modulus. Adding $<10 \mathrm{wt} \%$ PH11 to PH21 almost doubles the stress at yield. However, yield stress remains basically constant at $\sim 4 \mathrm{MPa}$ with a further increasing of the content of PH11. All blends show ill-defined yield points which become broader as the content of $\mathrm{PH} 21$ increases in the blends to the point that no clear yield point is observed for PH21. The higher and more distinct yield point of PH11-rich blends indicates that crystallites of PH11 are more resistant against load compared to the trigonal crystals of PH21, although the latter has thicker crystallites.

The correlation between yield stress and the lamellae thickness was studied for neat PH copolymers with different contents of 1-hexene [32]. Below $11 \mathrm{~mol} \%$ 1-hexene, where the copolymers crystallize in monoclinic phase, the yield strength decreases linearly with decreasing lamellae thickness (increasing 1-hexene content) [32]. The decreasing trend of yield stress with lamellae thickness is inverted at comonomer contents $>11 \mathrm{~mol} \%$ where a thicker trigonal phase is enabled, yet the yield stress continues decreasing [32]. The data of pure PH11 and pure PH21 follow the same trend. Moreover, the average lamellae thickness of the blends increases with increasing PH21, but as seen in Figure 3b, the yield stress is as high as for PH11 and basically independent of the lamellae thickness. Consequently, yielding in PH11/PH21 blends is governed by the presence of monoclinic PH11 crystallites. A higher yield stress of monoclinic PH11 compared to trigonal PH21 crystals of equal or higher thicknesses was explained by De Rosa and coworkers as driven by differences in the activation energy for crystallographic slips that are associated with the onset of yielding [32]. Due to the incorporation of 1-hexene units, the lamellae of defective trigonal crystals may be more prone to undergo crystallographic slippage than the crystals from the $\alpha$ phase that are less defective and are free of the 1-hexene units. 
Consequently, even small contents of PH11 crystals in the $\alpha$ phase will increase substantially the yield stress of the blends as seen in Figure $3 \mathrm{~b}$.

The plastic region is characterized by a decrease in resistance of crystals against the applied force. For the blends, the stress in this region does not change very much with elongation (Figure 2 and SI.1). The strain-softening region ranges from $\varepsilon \sim 15$ to $\varepsilon \sim 205 \%$ for $\mathrm{PH} 21$ and from $\varepsilon \approx 25 \%$ to $\varepsilon \approx 80 \%$ for $\mathrm{PH} 11$. In the blends, the width of the strain softening region is intermediate between the two components, and increases with increasing content of PH21. As mentioned, melt-recrystallization is a plausible mechanism in this region [38-41]. The molecules of partially molten crystals of PH11 after yielding can reorganize at relatively low strains into oriented crystals in the load direction. Melting and recrystallization takes place over a wider range of strain in the blends as the content of 1-hexene increases and the recrystallization kinetics become slower. Consequently, the strain hardening behavior of $\mathrm{PH} 21$-rich blends starts at higher elongations.

As shown in Figure 2, pure components and blends show high deformability $(\varepsilon>600 \%)$ and relatively high ultimate tensile strength $\left(\sigma_{b}>20 \mathrm{MPa}\right)$. The values of elongation at break $\varepsilon_{b}$, and the tensile stress at break, $\sigma_{b}$, are given in Figures $3 \mathrm{c}$ and $3 \mathrm{~d}$ as a function of PH11 content. A gradual small increase is observed for both, $\varepsilon$, and $\sigma_{b}$ with increasing content of PH11. The region of strain $>300 \%$ is of interest because pure components and blends show profound strain hardening, or a steep increase of stress with small changes in strain. To quantify the magnitude of strain hardening with blend composition, the difference $\sigma_{b}-\sigma_{y}$ is plotted vs. content of PH11 in Figure 3e.

The difference $\sigma_{b}-\sigma_{y}$ increases from about $15 \mathrm{MPa}$ to $30 \mathrm{MPa}$ with decreasing 1-hexene content, representing an increase in strain hardening as the content of PH11 increases in the blends. For the pure $\mathrm{PH}$ copolymers, $\sigma_{b}-\sigma_{\mathrm{y}}$ increases from about -4 to $15 \mathrm{MPa}$ with increasing 1-hexene content from 0 to $11.2 \mathrm{~mol} \%$ and then decreases with further increasing of 1-hexene content [32]. The latter is the same decreasing strain hardening trend observed in Figure 3e with increasing PH21 content in the blends. This trend is unchanged if the slope in the strain region between 550 and $700 \mathrm{MPa}$ (figure 2) is considered as representative of strain-hardening. Because the initial monoclinic crystals transform to a mesomorphic structure after yield, the variation of 
strain hardening with blend composition must be related with this transformation and with the development of strain-induced trigonal crystallites. These are oriented trigonal crystallites that serve as anchors between the mesophase and initial trigonal crystallites from the PH 21 component that do not undergo polymorphic transformation.

The elastomeric behavior of pure components and blends was measured by the recovery of the deformation after break, $r_{b}$, or their tendency to shrink back to their original length upon removing the load. $r \mathrm{~b}$ is plotted in Figure $3 \mathrm{f}$ as a function of blend composition. PH2 1 shows the highest elastomeric behavior upon removing the load with $r \mathrm{~b}>250 \%$, which represents close to one-third recovery after break. The elastic recovery of the blends decreases quickly with addition of PH1 1 and levels off to $\sim 120 \%$ for blends with $>50 \%$ PH11. The higher elastic recovery of PH21-rich blends is explained by a loss of orientation of trigonal crystals as it will be discussed later.

Polymorphic transformations under deformation: Prior to discussing WAXD patterns under deformation, the three major polymorphs pertinent to blends PH11/PH21 are shown in Figures 4 a-c with their corresponding crystallographic planes as a reference to further facilitate the discussion of polymorphic transformations under tensile deformation. The two broad peaks characteristic of the mesophase appear at $2 \theta=15^{\circ}$ and $21^{\circ}$ (Figure $4 \mathrm{c}$ ). 

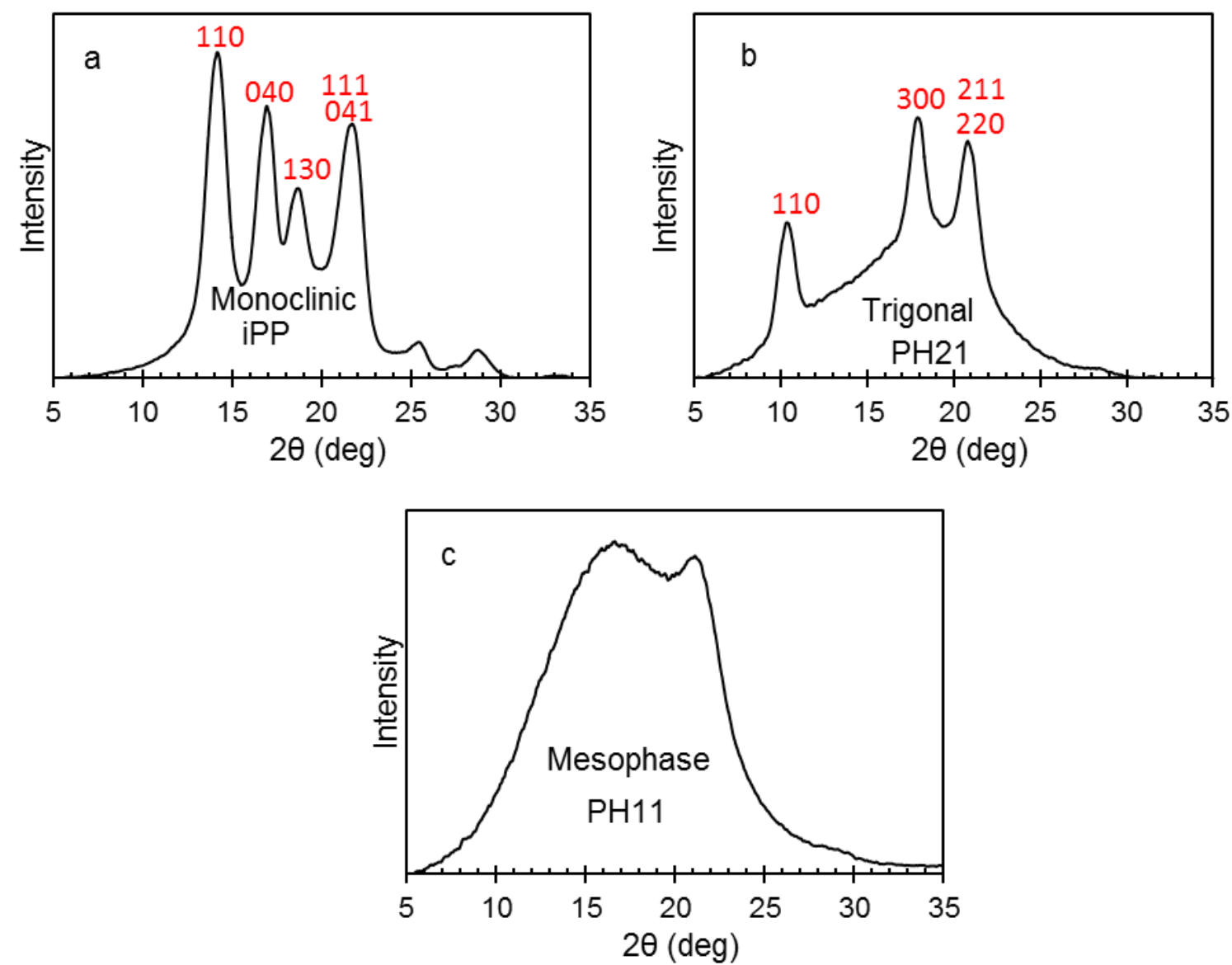

Figure 4. WAXD patterns and crystallographic planes corresponding to (a) monoclinic iPP, (b) trigonal $\mathrm{PH} 21,(\mathrm{c})$ mesophase formed in $\mathrm{PH} 11$ rapidly cooled to $0^{\circ} \mathrm{C}$.

Prior studies by De Rosa et al. have demonstrated that PH copolymers undergo a polymorphic transformation during tensile deformation [32]. Furthermore, it has been suggested that their high deformability and ultimate tensile strength are associated with crystal transformations during deformation [32]. Monoclinic PH copolymers with $<9$ mol\% 1-hexene transform to mesophase at elongations $100-200 \%$. At the highest elongation $(\sim 600 \%)$ pure mesophase were obtained for PH copolymers with contents of 1 - hexene in the range of 2.5 to 9 mol\% [32]. Copolymers with 1-hexene content between 9 and $18 \mathrm{~mol} \%$ display the same monoclinic to mesophase transformation and develop trigonal crystallites at deformations $>\sim 200 \%$ as indicated by the appearance of the 110 reflection at $2 \theta=10.5^{\circ}$, which is characteristic of the trigonal form [32]. PH copolymers with $>18 \mathrm{~mol} \%$ 1-hexene, which crystallize exclusively in trigonal phase, did not 
show any type of polymorphic transformation under uniaxial tensile deformation and only undergo orientation of the trigonal crystallites [32].

To correlate polymorphic transitions with the tensile behavior of PH11/PH21 blends, the specimens were stretched to a certain strain and WAXD/SAXS patterns were collected while the samples were kept under tension. 2D WAXD patterns collected for the initial unstretched specimens and at different levels of strain are shown in Figure 5. The 1D WAXD patterns integrated along the equatorial (perpendicular to load direction) in the azimuthal region between $75^{\circ}$ and $105^{\circ}$ are given in Figure 6. The azimuthal range used for integration is illustrated on the 2D WAXS of PH1 1 at $\varepsilon=620 \%$.

The behavior of the pure copolymers with increasing strain is akin to the behavior of analogs $\mathrm{PH}$ copolymers previously studied [32]. It is clear in Figure 5 that trigonal crystals of PH21 become highly oriented at $\varepsilon>200 \%$ and remain untransformed till fracture at $\varepsilon>700 \%$. The high orientation is indicated by point-like reflections at $2 \theta=10.5^{\circ}, 17.7^{\circ}, 20.6^{\circ}$ and $20.8^{\circ}$ corresponding respectively to planes $(110)_{\delta},(300)_{\delta},(220)_{\delta}$ and $(211)_{\delta}[18,20]$. The 1D WAXD patterns of PH21 (Figure 6) indicate that with increasing strain the intensity (area) of the $(110)_{\delta}$ reflection increases pointing that in addition to orientation, strain-induced trigonal crystallization occurs at high elongation. A similar behavior was observed by De Rosa et al. for $\mathrm{PH}$ copolymer with $>18 \mathrm{~mol} \%$ 1-hexene under deformation [32].

Pure PH11 shows more complex polymorphic transformations under deformation. At room temperature this polymer forms a mixture of defective monoclinic crystals coexisting with a disordered mesophase structure (last panel of Figures 5 and 6). Orientation develops shortly after yield, at $\varepsilon \sim 100 \%$, and is coupled with transformation of the monoclinic to mesophase as shown in the 2D WAXD patterns by a broad reflection in the equator. Weak isotropic rings indicative of the original random lamellae are still observed at this point. The isotropic rings disappear at elongations $>250 \%$ indicating a complete disruption of the initial crystal structure. Interestingly, at the beginning of strain-hardening, $\varepsilon \sim 250 \%$, the characteristic spot of the (110) plane of the trigonal phase appears in the equator and becomes better defined at higher elongations. This new equatorial reflection is a clear indication of development of oriented trigonal crystallites, also shown in the 1D WAXD profile of Figure 6 by the reflection at $10.5^{\circ}$ characteristic of the 
trigonal phase. This reflection is absent in undeformed PH11.
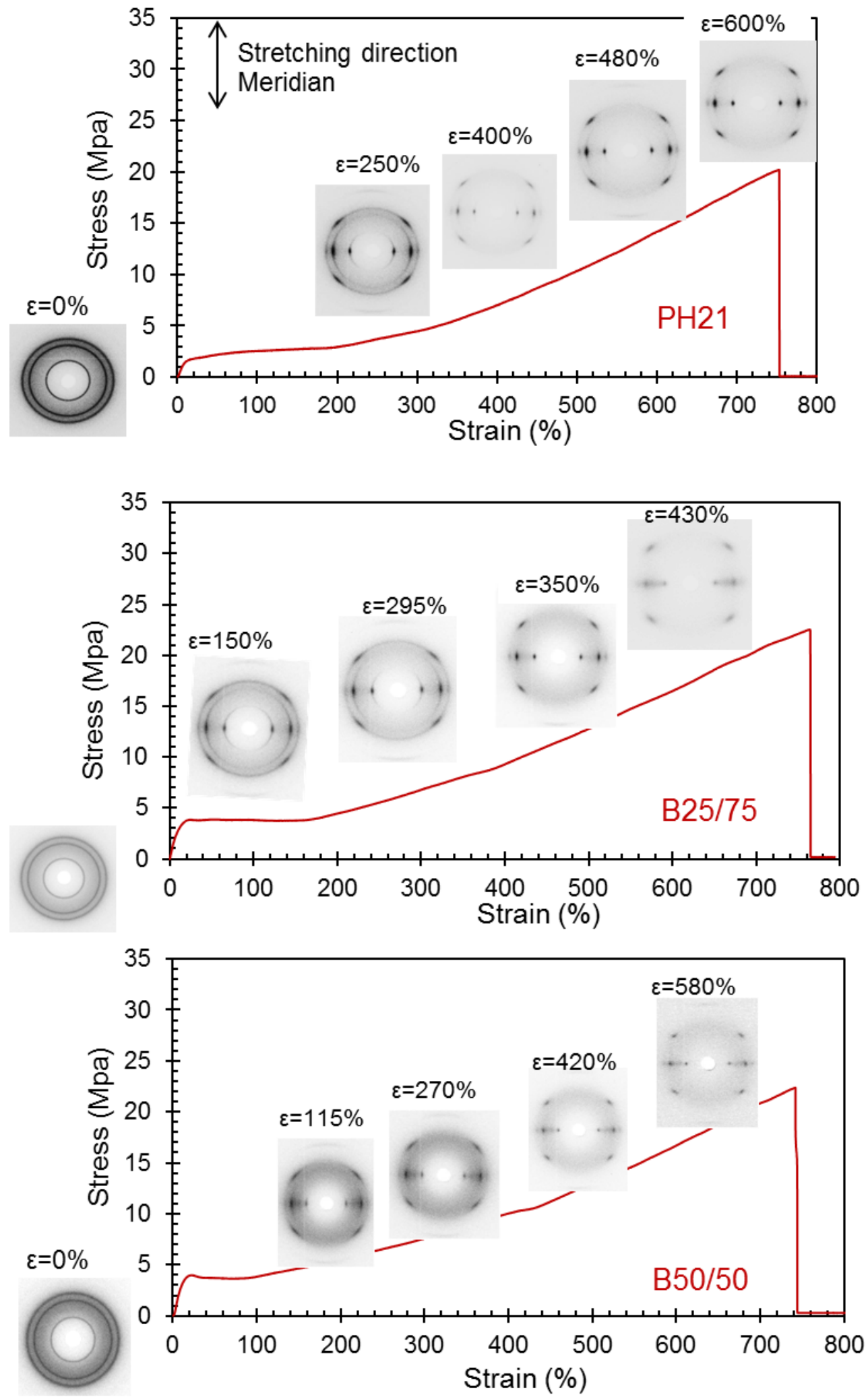

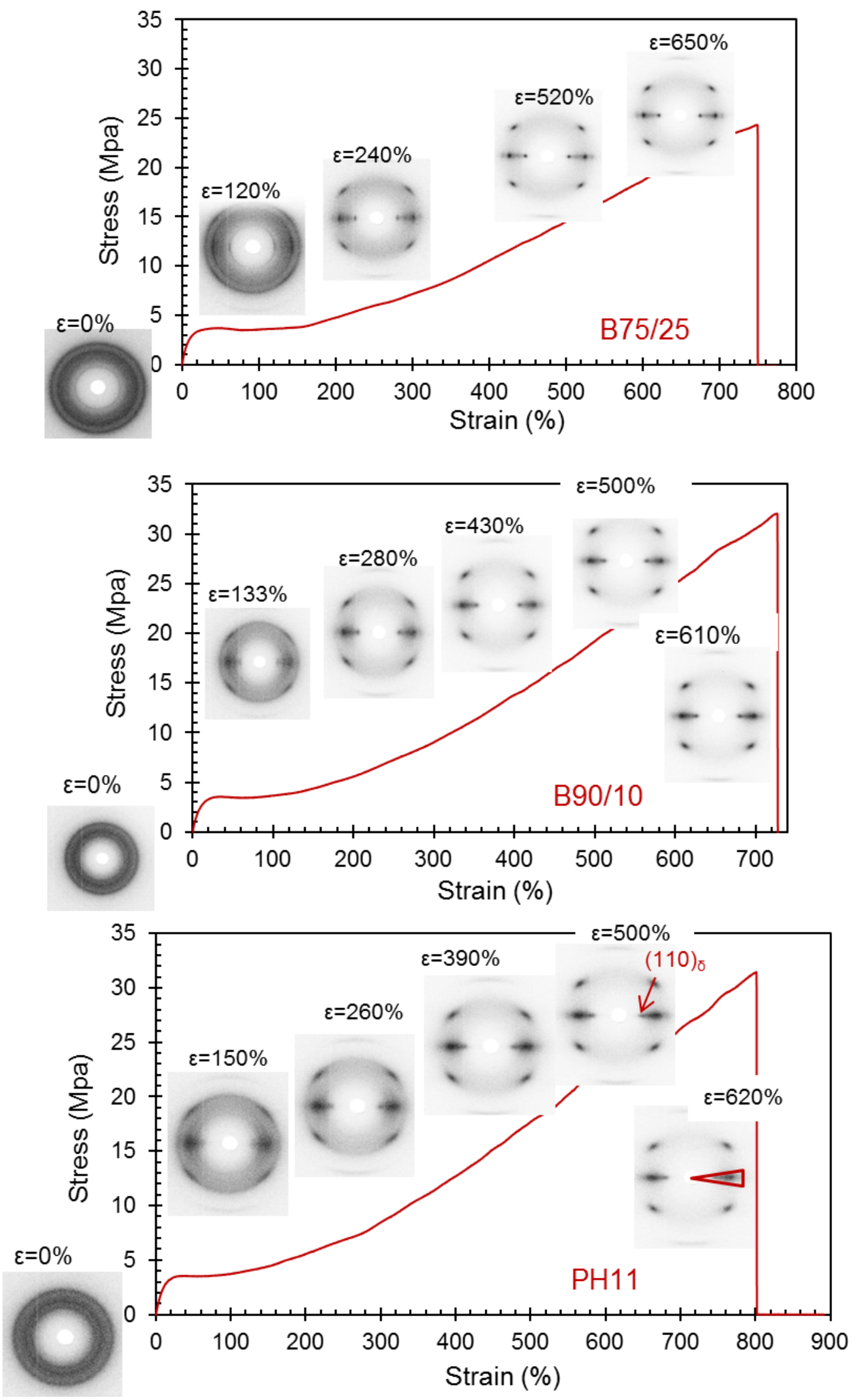
Figure 5. Stress-strain curves and 2D WAXD patterns collected at different elongations. The triangle on the 2D WAXD pattern of PH11 at $\varepsilon=620 \%$ shows the azimuthal range of integration used for $1 \mathrm{D}$

WAXD patterns.

The behavior of the blends shows the characteristic transformation and orientation from both components. The initial underformed blends crystallize in a mixture of monoclinic and trigonal crystals except for B90/10 that does not show any detectable trigonal peak in the WAXD pattern at $\varepsilon=0 \%$ (Figure 5). After the yield point, orientation and transformation of monoclinic crystals to mesophase are observed for all blends starting in the strain-softening region as indicated by the transformation of reflections corresponding to (040) and (130) planes of the monoclinic phase into a broad spot at the equator. However, in the strain-softening region, the trigonal crystals still maintain a predominant random distribution with minor orientation inferred from arc-shaped reflections of plane (110) in B10/90, B25/75, B75/25 (Figure 5). At higher strains (in the strain hardening region), the arc-shaped reflections of the trigonal transform into intense dots in the equatorial direction, indicative of a highly oriented structure. The intensity of the reflections at the equator increases as the breaking point is approached. With increasing content of PH11, the reflections at the equator become more diffuse due to the monoclinic to mesophase transformation and the overlapping of broad mesophase reflection at $2 \theta=16-18^{\circ}$ with the trigonal reflection at $2 \theta=17.7^{\circ}$. 

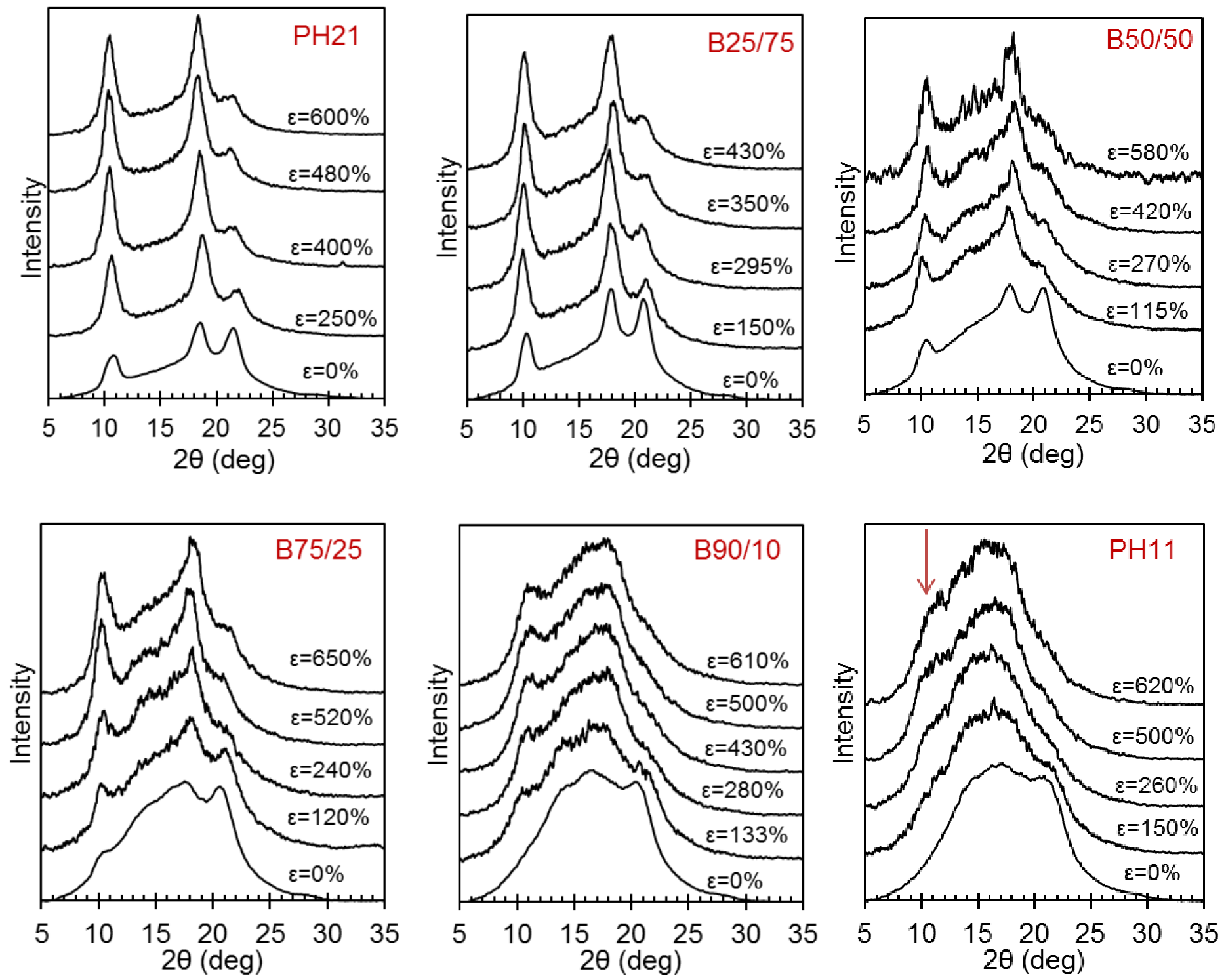

Figure 6. 1D WAXD patterns of PH11, PH21 and blends of PH11/PH21 integrated in the azimuthal angle between $75^{\circ}$ to $105^{\circ}$. For non-stretched samples with random distribution of crystallites integration is between $0^{\circ}$ to $360^{\circ}$.

In order to quantify the change in content of trigonal crystallites with increasing elongation, the ratio of the area under peak at $2 \theta=10.5^{\circ}$ to the total area of the halo-subtracted diffractogram (Figure 7a) is taken as a trigonal crystallinity factor, ftrigonal, or a parameter associated with content of trigonal form. ftrigonal versus strain for pure components (Figure $7 \mathrm{~b}$ ) and blends (Figure 7c) displays a rapid increase at the beginning of the strain hardening region and levels off beyond $\varepsilon>\sim 200 \%$. 
The formation of trigonal crystallites for PH11 and B90/10 under deformation is of interest because trigonal crystals are absent when these samples are crystallized from quiescent melts. Hence, the formation of the trigonal phase under tensile deformation must be related to the increase in 1-hexene content in the intercrystalline region and the decrease in entropy of this region upon orientation. To analyze these changes, we focus first in the difference in isotactic sequence length distribution (ISLD) between the intercrystalline region of crystallized PH11 and the molten PH11. Then, we analyze how chain orientation could facilitate the formation of trigonal crystallites.

The ISLD of the intercrystalline regions of PH11 was computed according to $W_{n}-n\left(1-X_{A}\right)^{2} X_{A}^{n-1}$. Here, $W_{\mathrm{n}}$ is the fractional content of sequences of length $n$, and $X_{\mathrm{A}}$ the fraction of crystalline units. The fraction of crystalline propene units in the intercrystalline region $\left(X_{\mathrm{A}}\right)$ was estimated from the level of crystallinity obtained from WAXD, as detailed in a prior work [30]. ISLD for molten PH11, molten PH21 and in the amorphous regions of PH11 crystallized at $30^{\circ} \mathrm{C}$ are given in Figure 8 . The shaded area in the figure represents the content of short sequences $(3<n<7)$ responsible for formation of the trigonal phase. Because the 1-hexene comonomer is excluded from the PH11 crystals, after crystallization, the concentration of short sequences in the amorphous regions increases considerably, even at levels higher than for PH21 as seen in this figure; yet trigonal crystals do not form for PH11. Confinement of the short sequences in the intercrystalline region most likely restricts diffusion to form trigonal crystallites. Furthermore, the crystallization kinetics of this phase is very slow [30]. However, upon elongation and beyond the yield point, the partial melting and transformation of monoclinic crystallites to the mesophase coupled with orientation in the direction of elongation changes the entropy of the molecules of the intercrystalline region where the short sequences are more accumulated. The decrease in entropy of this region facilitates the formation of trigonal crystals. Therefore, the rationale for the formation of trigonal crystals on elongation in the blends, including PH11 and B90/10 that do not form this phase from quiescent melts, is a combination of increased comonomer concentration in the intercrystalline regions and orientation to decrease the entropy barrier for nucleation of this phase. 

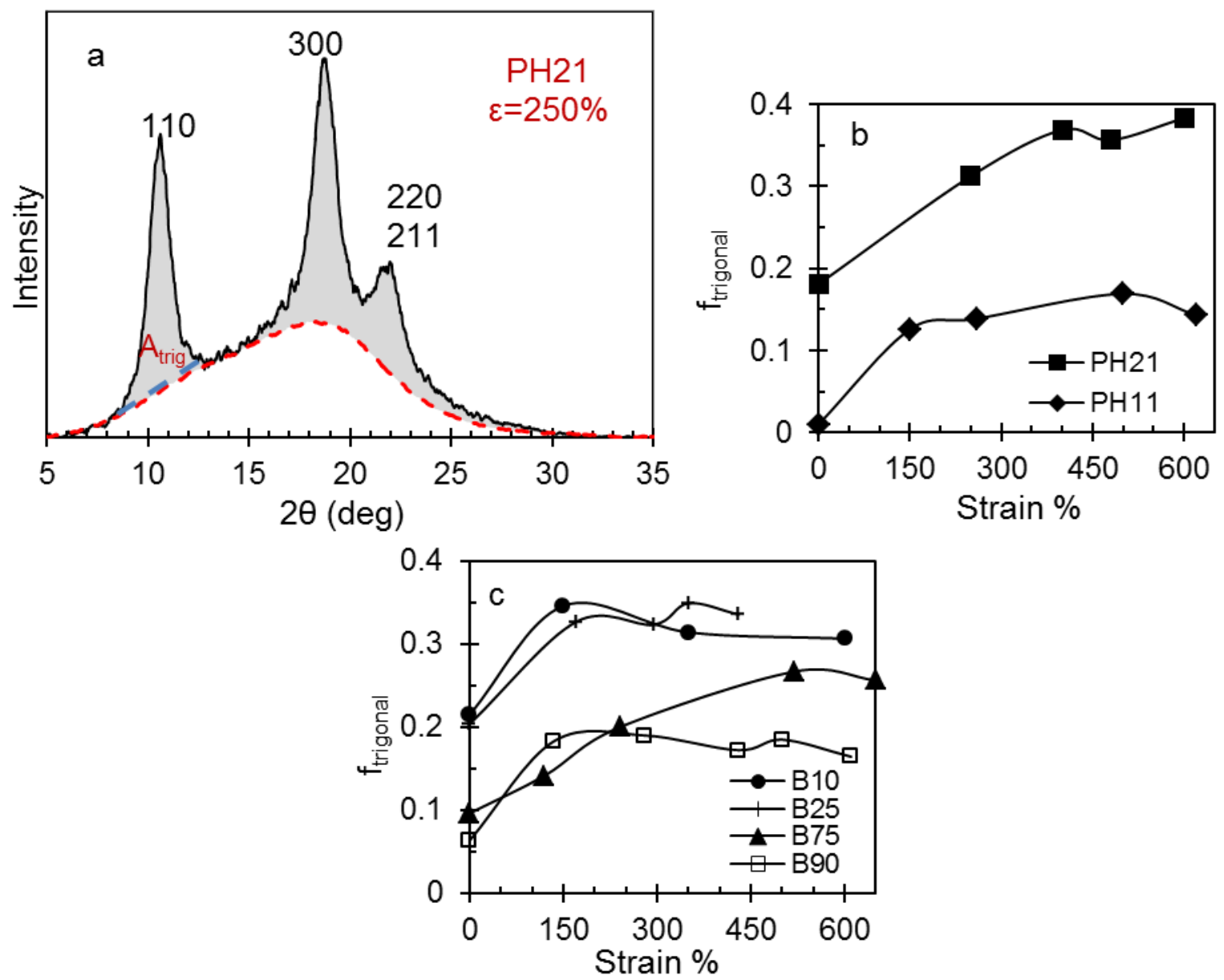

Figure 7. (a) Estimation of trigonal factor from WAXD patterns of pure components and blends. Trigonal factor vs. elongation for (b) PH11 and PH21, (c) PH11/PH21 blends.

The content of 1-hexene in the intercrystalline regions of the blends was calculated taking into account the two step crystallization, and the levels of crystallinity corresponding to each step. In the first step of formation of monoclinic crystals, the content of 1-hexene in the surrounding melt is calculated as for pure PH11 with the appropriate crystallinity level after the first step [30]. The formation of trigonal crystals in the second step leaves a final fraction of 1-hexene in the

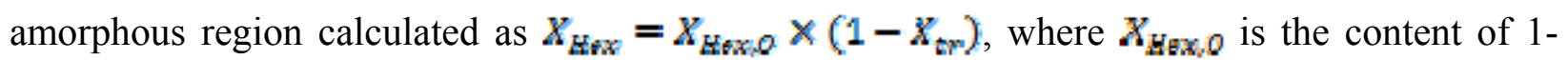
hexene in the melt at the end of first step, and $X_{\mathrm{s}}$ is trigonal crystallinity obtained from WAXD [30]. 


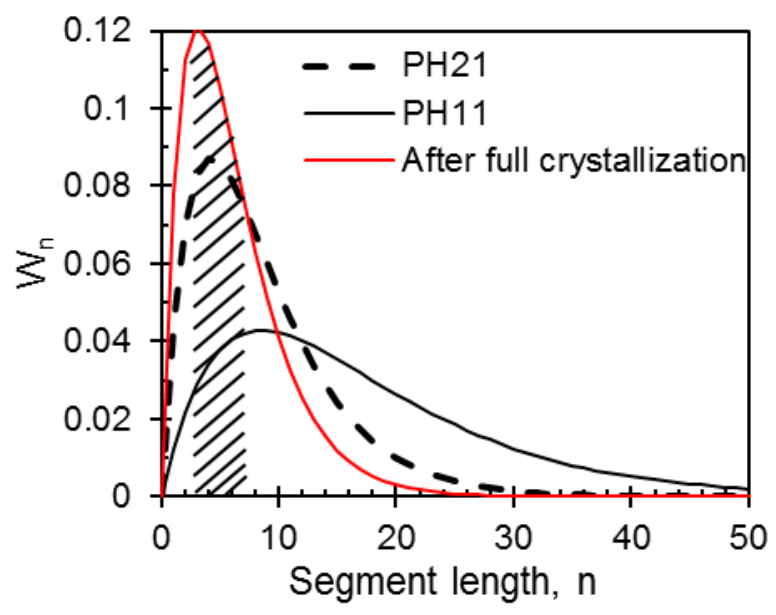

Figure 8. Isotactic sequence length distribution (ISLD) of molten PH11 and in amorphous regions after crystallization compared with ISLD of molten PH21. The shaded area shows the content of isotactic sequences with length 3 to 7 repeat units.

Figure 9 shows the content of 1-hexene in the melt and values calculated for the intercrystalline regions as a function of blend composition. The horizontal dotted lines demarcate the region of 1-hexene content for the formation of the trigonal form [30]. As shown in this figure, in all nonstretched samples crystallized at room temperature the content of 1-hexene in the amorphous region is sufficient for further formation of trigonal phase. Hence, on tensile elongation, all blends must undergo stress-induced trigonal crystallization as shown by a large increase of the trigonal factor after the monoclinic to mesophase transformation (Figure 7). Consequently, the polymorphic transformation coupled with further stress induced crystallization, allows better stress transfer and makes the blends hold to high levels of stress and orientation even after high deformations.

The formation of trigonal phase in PH11 under deformation is independent of initial crystal structure since it was also observed in a PH11 that was rapidly crystallized at low temperature to develop only the mesomorphic form (supplementary Figure SI.2). In the case of mesomorphic PH11 the characteristic peak of trigonal appears at elongations above $\sim 250 \%$ while for monoclinic PH11 it appears at $\sim 150 \%$. Similarly, the intensity of the peak at $2 \theta=10.5^{\circ}$ increases with strain indicating the increase in orientation as well the increase in content of trigonal crystals. 


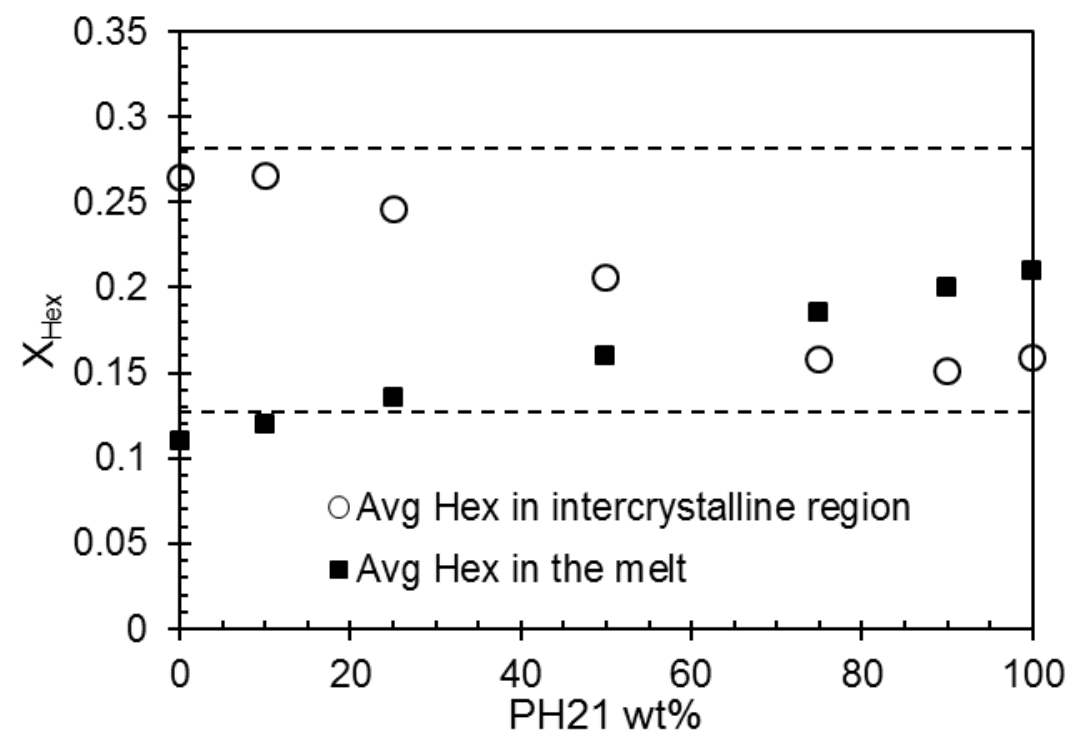

Figure 9. Average content of 1-hexene in the melt (filled squares) and in intercrystalline regions after full crystallization (open circles), as a function of content of PH21 in the blends.

The polymorphic transformation coupled with orientation and stress-induced crystallization of the trigonal phase of pure components and PH11/PH21 blends are schematically summarized in Figure 10. Lamellae and oriented fibrils of trigonal crystals are represented in green, monoclinic crystals as blue, and stretched-induced mesophase in red. In the axialitic structure of $\mathrm{PH} 21$, at $\varepsilon=0 \%$, lamellar trigonal crystals are randomly distributed [14]. With increasing elongation $(0 \%<\varepsilon<100 \%)$, the amorphous phase is stretched and the load is transferred through tie molecules to the lamellae. Consequently, the lamellar stacks of trigonal phase partially melt and rotate and rotated to align the c-axis of the lamellae stems with the direction of load. After yield and at higher elongations ( $\varepsilon>100-150 \%)$ the original lamellar structure becomes fragmented and further orients into trigonal fibrils (green oriented lines) in the strain hardening region, $\varepsilon>200 \%$. Conversely, in PH11, a mixture of monoclinic lamellae (blue) and mesophase (red objects) exists in non-stretched samples. Due to efficient stress transfer the original lamellar stacks of monoclinic crystals transform into mesomorphic fibrils with lateral disorder at relatively low strains $(50 \%<\varepsilon<100 \%)$ as shown by a decrease in the content of blue crystals and increase in the content of oriented red lines (stress-induced mesophase). At elongations $>200 \%$, the entropy of the system has decreased sufficiently that short sequences can overcome the free energy barrier for nucleation and form oriented trigonal fibrils (green objects in the figure). The deformation of the blends shows the characteristics of PH11 and PH21. A mixture of trigonal and monoclinic 
lamellae exists in the blends at $\varepsilon=0 \%$. At $50 \%<\varepsilon<100 \%$, the $\alpha$-mesophase transition occurs in the blends, at this point the trigonal lamellae are slightly aligned to the direction of load. The fibrillar structure of long sequences of PH11 at elongations $100 \%<\varepsilon<250 \%$, enables short isotactic sequences to be suitable for trigonal formation by the decrease in entropy in the interfibrillar regions. These strain-induced trigonal crystals are surrounded by mesomorphic and some monoclinic crystals which act as stabilizers for strain-induced trigonal crystals. It is also observed that as the content of the stabilizer (monoclinic fibrils) increases in blends, the melting temperature of oriented trigonal crystals also increases (Figure SI.3).

\section{$\mathrm{PH} 21$}
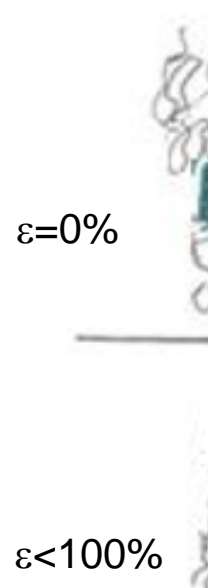

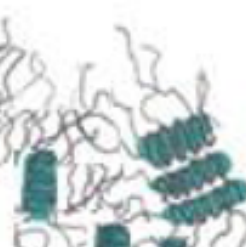

S 8

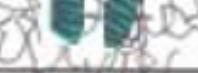

$\mathrm{PH} 11$

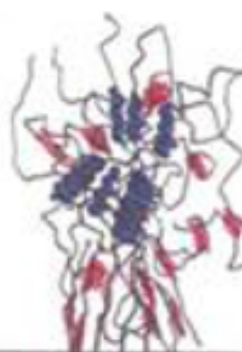

\section{$\mathrm{PH} 11 / \mathrm{PH} 21$}

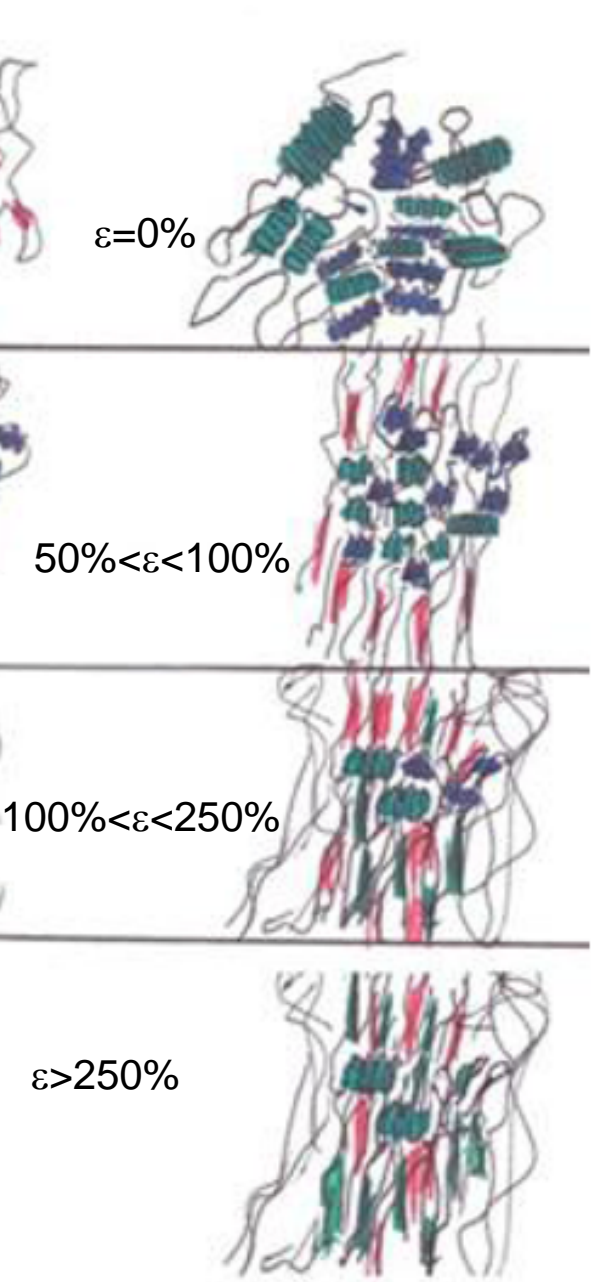


Figure 10. Schematics of structure under deformation for pure PH21, PH11 and a typical blend of $\mathrm{PH} 11 / \mathrm{PH} 21$.

Lastly, we investigated if oriented stretched-induced trigonal crystals are stable at room temperature after breaking. 2D WAXD patterns of PH11, PH21 and representative blends are shown in Figure 11 for the initial films, the films under high elongation $(>600 \%)$, and 2 days after breaking. For the latter, WAXD patterns were taken in a region close to the breaking line. The differences are quite obvious; the initial random orientation of $\mathrm{PH} 21$ is almost fully recovered indicating that the lamellar to fibrillary transformation during deformation of $\mathrm{PH} 21$ is reversible upon removing the load. Moreover, orientation remains in all blends, more so with increasing content of PH11 as indicated by the persistence of high intensity spots at the equator. Because the content of stress-induced trigonal crystals in the intercrystalline regions increase with increasing PH11, these crystals act as physical crosslinks preventing the molecules of $\mathrm{PH} 21$ to relax to the random orientation.

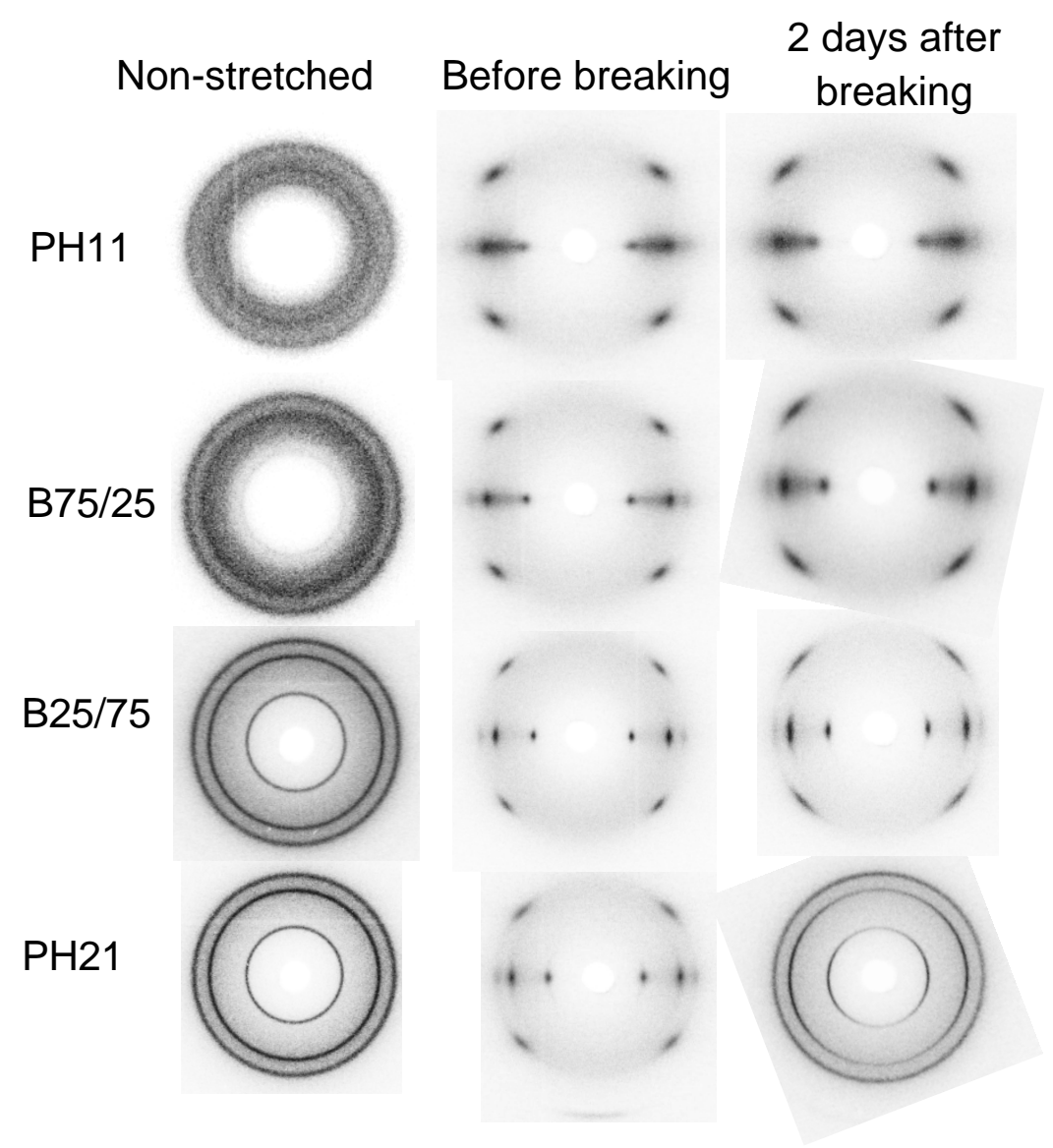


Figure 11. 2D WAXD diffractograms of PH11 and PH21 and their blends under tensile stress, before and after breaking.

\section{Conclusions}

The mechanical properties under uniaxial tensile deformation and associated polymorphic transitions followed by WAXD of pure components and blends of two propylene 1-hexene copolymers with 11 and 21 mol\% comonomer (PH11 and PH21) were studied. Both polymers are thermoplastic elastomers with characteristic stress-strain profiles in four distinct regions: linear elastic region, yield point, strain softening $\left(\frac{d q}{d z} \approx 0\right)$, and strain hardening. Values for the elastic modulus and yield strength do not change significantly with blend composition because both copolymers and blends have similar crystallinity levels. Monoclinic crystals of PH11 show higher resistance to external load compared to trigonal crystallites of $\mathrm{PH} 21$, a feature reflected in the moduli of the blends even at low content of PH11. The tensile properties after yield in the strain-softening and strain-hardening regions are determined mainly by the reinforcing effect of stress-induced crystallization of trigonal crystallites with PH11 showing the narrowest strain softening region and highest strain hardening behavior due to a faster kinetics of re-organization under deformation. Stress-induced formation of trigonal crystallites is explained by the entropic decrease of the oriented interfibrillar regions and a relatively high concentration of short isotactic sequences in these regions as indicated by the increase of trigonal content with degree of orientation. The transformation of monoclinic to mesophase after yield in PH11 and all blends stabilizes the oriented stress-induced trigonal crystallites such that a high degree of orientation remains after removal of load and after break. Schematics are offered for crystal transformations during elongation of PH11, PH21 and their blends.

\section{Acknowledgments}

This material is based upon work supported by the National Science Foundation under Grant DMR1105129. We acknowledge the FSU High Performance Materials Institute (HPMI) for access to the x-ray diffractometer and Prof. J. Schlenoff for access to tensile instrumentation. 


\section{References}

[1] De Rosa C, Auriemma F, Ruiz de Ballesteros O, Resconi L, Camurati I. Tailoring the physical properties of isotactic polypropylene through incorporation of comonomers and the precise control of stereo and regioregularity by metallocene catalysts. Chem. Mater. 2007;19:5122-30.

[2] De Rosa C, Auriemma F, Di Capua A, Resconi L, Guidotti S, Camurati I, Nifantev IE, Laishevtsev IP. Structure-Property Correlations in Polypropylene from Metallocene Catalysts: Stereodefective, Regioregular Isotactic Polypropylene J. Am. Chem. Soc 2004;126:17040-49

[3] De Rosa C, Auriemma F. The Deformability of Polymers: Structural-Mechanical Phase Diagram of Isotactic Polypropylene. J. Am. Chem. Soc. 2006;128:11024-11025

[4] De Rosa C, Auriemma F, Di Girolamo R, Ruiz de Ballesteros O, Pepe M, Tarallo O, Malafronte A. Morphology and Mechanical Properties of the Mesomorphic Form of Isotactic Polypropylene in Stereodefective Polypropylene Macromolecules 2013;46:5202-5214

[5] Toki S, Sics I, Burger C, Fang D, Liu L, Hsiao BS, Datta S, Tsou AH. Structure Evolution during Cyclic Deformation of an Elastic Propylene-Based Ethylene-Propylene Copolymer. Macromolecules 2006;39:3588-97

[6] Iizuka Y, Sugiyama J, Hagihara H. Unexpected Mechanical Properties of Functionalized Polypropylene: Tensile Test, Charpy Impact Tensile Test, DSC, and WAXD Analysis of Poly(5-hexen-1-ol-co-propylene). Macromolecules 2009;42:2321-23

[7] Menyhárd A, Suba P, László Zs, Fekete HM, Mester ÁO, Horváth Zs, Vörös Gy, Varga J, Móczó J. Direct correlation between modulus and the crystalline structure in isotactic polypropylene. eXPRESS Polym. Let. 2015;9:308-320

[8] Arranz-Andres J, Suarez I, Pena B, Benavente R, Perez E, Cerrada ML. Metallocenic Isotactic Poly(propylene) and its Copolymers with 1-Hexene and Ethylene. Macromol. Chem. Phys. 2007, 208, 1510-21

[9] Shi X, Tang , Li Y-s. Random copolymers of propylene with 1,5-hexadiene containing only cyclopentane units in main chain and tailoring structure and mechanical properties of the copolymers. Polymer 2011;52:3053-58

[10] Mehta AK, Chen MC, McAlpin JJ. In: Benedikt GM, Goodall BL, editors. Metallocenecatalyzed polymers: materials, properties, processing and market. New York: Plastic Design Library; 1998. p. 261.

[11] Alamo RG, Kim M-H, Galante MJ, Isasi JR, Mandelkern L. Structural and Kinetic Factors Governing the Formation of the $\gamma$ Polymorph of Isotactic Polypropylene. Macromolecules 1999;32:4050-64. 
[12] Hosier IL, Alamo RG, Esteso P, Isasi JR, Mandelkern L. Formation of the $\alpha$ and $\gamma$ Polymorphs in Random Metallocene-Propylene Copolymers. Effect of Concentration and Type of Comonomer. Macromolecules 2003;36:5623-36. Hosier, I L, Alamo RG, Lin, JS. Polymer 2004;45:3441. Alamo RG, Ghosal A, Chatterjee J, Thompson KL. Polymer 2005;46:8774.

[13] Ruiz-Orta C, Fernandez-Blazquez JP, Anderson-Wile AM, Coates GW, Alamo RG. Isotactic Polypropylene with $(3,1)$ Chain-Walking Defects: Characterization, Crystallization, and Melting Behaviors. Macromolecules 2011;44:3436-51

[14] Jeon K, Palza H, Quijada R, Alamo RG. Effect of comonomer type on the crystallization kinetics and crystalline structure of random isotactic propylene 1-alkene copolymers. Polymer 2009;50:832.

[15] Jeon K, Chiari YL, Alamo RG. Maximum Rate of Crystallization and Morphology of Random Propylene Ethylene Copolymers as a Function of Comonomer Content up to $21 \mathrm{~mol} \%$ Macromolecules 2008;41:95-108.

[16] Arnold M, Henschke O, Knorr J. Copolymerization of propene and higher $\alpha$-olefins with the metallocene catalyst Et[Ind $]_{2} \mathrm{HfCl}_{2}$ /methylaluminoxane. Macromol. Chem. Phys. 1996;197:563. Arnold M, Bornemann S, Koller F, Menke TJ, Kressler J. Macromol. Chem. Phys.

1998;199:2647. Busse K, Kressler J, Maier RD, Scherble J. Macromolecules 2000;33:8775.

[17] Shin Y.-W, Uozumi T, Terano M, Nitta K.-H. Synthesis and characterization of ethylenepropylene random copolymers with isotactic propylene sequence. Polymer 2001;42:9611. Shin Y.-W, Hashiguchi H, Terano M, Nitta, K. J. Appl. Polym. Sci. 2004;92:2949.

[18] Lotz B, Ruan J, Thierry A, Alfonso GC, Hiltner A, Baer E, Piorkowska E, Galeski A, Structure of Copolymers of Propene and Hexene Isomorphous to Isotactic Poly(1-butene) Form I. Macromolecules 2006;39:5777-81

[19] Poon B, Rogunova M, Hiltner A, Baer E, Chum S, Galeski A, Piorkowska E. Structure and properties of homogeneous copolymers of propylene and 1-hexene. Macromolecules, 2005;38:1232-43.

[20] De Rosa C, Auriemma F, Corradini P, Tarallo O, Dello Iacono S, Ciaccia E, Resconi L. Crystal Structure of the Trigonal Form of Isotactic Polypropylene as an Example of DensityDriven Polymer Structure L. J. Am. Chem. Soc. 2006;128:80-81.

[21] De Rosa C, Auriemma F, Talarico G, Ruiz de Ballesteros O. Structure of Isotactic Propylene-Pentene Copolymers Macromolecules 2007;40:8531-8532 
[22] De Rosa C, Auriemma F, Talarico G, Ruiz de Ballesteros O. The Double Role of Comonomers on the Crystallization Behavior of Isotactic Polypropylene: Propylene-Hexene Copolymers Macromolecules 2008;41:2172-2177

[23] De Rosa C, Auriemma F, Ruiz de Ballesteros O, Resconi L, Camurati I. Crystallization Behavior of Isotactic Propylene-Ethylene and Propylene-Butene Copolymers: Effect of Comonomers versus Stereodefects on Crystallization Properties of Isotactic Polypropylene. Macromolecules, 2007;40:6600-16

[24] Palza H, Lopez-Majada JM, Quijada R, Benavente R, Perez E, Cerrada ML. Metallocenic Copolymers of Isotactic Propylene and 1-Octadecene: Crystalline Structure and Mechanical Behavior. Macromol. Chem. Phys. 2005;206:1221-30

[25] Bartczak Z, Pracella M. Blends of propylene-ran-ethylene and propylene-ran-(1-butene) copolymers: Crystal superstructure and mechanical properties. European Polym. J. 2006;42: 1819-29.

[26] Bartczak Z, Pracella M. Blends of propylene-ethylene and propylene-1-butene random copolymers: I. Morphology and structure. Polymer. 2004;45: 7549-61.

[27] Wang J, Niu H, Dong J, Du J, Han CC. Morphology and mechanical properties of polypropylene/poly

(propylene-1-octene) in-reactor alloys prepared by Metallocene/Ziegler-Natta hybrid catalyst. Polymer 2012;53:1507-16.

[28] Fu ZS, Fan ZQ, Zhang YQ, Feng LX. Structure and morphology of polypropylene / poly(ethylene-co-propylene) in situ blends synthesized by spherical Ziegler-Natta catalyst. Eur Polym J 2003;39:795-804.

[29] Janani H, and Alamo RG. Melt miscibility of blends of isotactic polypropylene and homogeneous iso-propylene-1-hexene copolymers. J. Therm. Anal. Calorim. 2014;116:14971508 .

[30] Janani H, Alamo RG. Enhanced rate of formation of trigonal phase in blends of homogenous isotactic propylene-1-hexene copolymers. Polymer 2015;64:163-175.

[31] De Rosa C, Dello Iacono S, Auriemma F, Ciaccia E, Resconi L. Crystal structure of isotactic propylene-hexene copolymers: the trigonal form of isotactic polypropylene. Macromolecules 2006;39:6098-109.

[32] De Rosa C, Auriemma F, de Ballesteros O, Iacono S. Stress-induced polymorphic transformations and mechanical properties of isotactic propylene-hexene copolymers. Crystal and growth design. 2009;9:165-76. 
[33] Lopez-Majada JM, Palza H, Guevara JL, Martinez MC, Benavente R, Perena JM, Perez E., Cerrada ML. Metallocene copolymers of propene and 1-hexene: The influence of comonomer content and thermal history on the structure and mechanical properties. J. Polym. Sci. Polym. Phys. 2006; 44: 1253-67.

[34] Santa Cruz C, Stribeck N, Zachmann HG, Blata FJ. Novel aspects in the structure of poly (ethylene terephthalate) as revealed by means of small angle x-ray scattering. Macromolecules 1991;24:5980-90.

[35] Kennedy MA, Peacock AJ, Mandelkern L. Tensile Properties of Crystalline Polymers: Linear Polyethylene. Macromolecules 1994;27:5297-5310.

[36] Bowden PB, Young RJ. Deformation mechanisms in crystalline polymers. J. Mater. Sci. 1974;9:2034-51.

[37] Young P, Stein RS, Kyu T. Static small-angle x-ray scattering study of polymer deformation. J. Polym. Sci., PartB: Polym. Phys. 1990; 28:1791-1812.

[38] Flory PJ, Yoon DY. Molecular morphology in semicrystalline polymers. Nature 1978;272:226-9.

[39] Popli R, Mandelkern L. Influence of Structural and Morphological factors on the Mechanical Properties of the Polyethylenes. J. Polym. Sci., Polym. Phys. Ed.1987;25:441.

[40] Mao Y, Li X, Burger C, Hsiao BS, Mehta A, Tsou A. Structure development during stretching and heating of isotactic propylene-1-butylene random copolymer: From unit cells to lamellae. Macromolecules 2012;45:7061-71.

[41] Cai Z, Zhang Y, Li J, Xue F, Shang Y, He X, Feng J, Wu Z, Jiang. Real time synchrotron SAXS and WAXS investigations on temperature related deformation and transitions of $\beta$-iPP with uniaxial stretching Polymer 2012;53:1593-1601.

[42] Crist B, Fischer CJ, Howard PR. Mechanical properties of model polyethylenes: tensile elastic modulus and yield stress. Macromolecules 1989;22:1709-18.

[43] Zhao J, Sun Y, Men Y. Elasticity Reinforcement in Propylene-Ethylene Random Copolymer Stretched at Elevated Temperature in Large Deformation Regime. Macromolecules 2016;49:609-615.

[44] Kang J, Yuan S, Hong Y, Chen W, Kamimura A, Otsubo A, Miyoshi T. Unfolding of Isotactic Polypropylene under Uniaxial Stretching. ACS Macro Lett. 2016;5:65-68.

[45] Young RJ, Bowden PB, Ritchie JM, Rider JG. Deformation mechanisms in oriented highdensity polyethylene. J.Mater. Sci. 1973;8:23-26.

[46] Peacock AJ, Mandelkern L. The mechanical properties of random copolymers of ethylene: Force-elongation relations. J. Polym. Sci. Polym. Phys. 1990;28:1917-41. 
[47] Sun Y, Fu L, Wu Z, Men Y. Structural Evolution of Ethylene-Octene Copolymers upon Stretching and Unloading Macromolecules. 2013;46:971-976. 